\title{
Comportamiento elástico de la madera de Acer rubrum y de Abies balsamea
}

\section{Elastic behavior of Acer rubrum and Abies balsamea wood}

\author{
Saúl Antonio Hernández-Maldonado' y Javier Ramón Sotomayor-Castellanos²
}

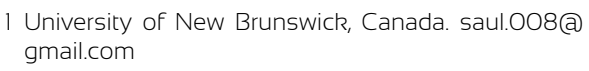

2 Universidad Michoacana de San Nicolás de Hidalgo. México. madera999@yahoo.com

\section{RESUMEN}

El diseño de productos con alto valor agregado y el cálculo de estructuras en madera requieren de características elásticas confiables y relacionadas con un modelo teórico el cual explique de manera racional el comportamiento elástico de la madera. Se presentan la metodología y los resultados de la determinación experimental de las características elásticas de dos maderas canadienses: Acer rubrum y Abies balsamea. Las características examinadas fueron: módulos de elasticidad, módulos de rigidez y coeficientes de Poisson. Se realizaron experimentos de compresión, en el dominio elástico, de las direcciones de ortotropía de la madera: radial, tangencial y longitudinal. El contenido de humedad fue de $9 \%$ para A. rubrum y de $10 \%$ para A. balsamea. Las densidades de las maderas fueron de $651 \mathrm{~kg} / \mathrm{m}^{3}$ y $393 \mathrm{~kg} / \mathrm{m}^{3}$ respectivamente. A partir de las características obtenidas empíricamente, se analizaron las propiedades y las relaciones de ortotropía de la matriz de constantes elásticas del modelo elástico general. Los valores experimentales de las características elásticas de las maderas de A. rubrum y de A. balsamea, son comparables con valores correspondientes a estas maderas determinados en otros estudios. Igualmente, se demostró que los postulados de ortotropía del modelo elástico general son -con cierto nivel de confianza- válidos.

PALABRAS Clave: coeficiente de Poisson, modelo elástico, módulo de elasticidad, módulo de rigidez, ortotropía.

\section{ABTRACT}

This design of high value-added products and the calculus of structures in wood requires reliable elastic characteristics related to a theoretical model that explains in a rational way the elastic behavior of wood. This research presents the methodology and the results of the experimental determination of the elastic characteristics of two Canadian woods: Acer rubrum and Abies balsamea. The characteristics examined were modulus of elasticity, modulus of rigidity and Poisson's ratio. Compression tests within the elastic regime were performed in the orthotropic directions of wood: radial tangential and longitudinal. The moisture content of $A$. rubrum was $9 \%$ and $10 \%$ for A. balsamea. The densities were $651 \mathrm{~kg} / \mathrm{m}^{3}$ and $393 \mathrm{~kg} / \mathrm{m}^{3}$ respectively. With the empirically obtained characteristics, the properties and orthotropic relations of the elastic constants matrix of the general elastic model were analyzed. The experimental values of the elastic characteristics of A. rubrum and of A. balsamea woods are comparable with values corresponding to these woods determined in other studies. Likewise, it was proved that the orthotropic postulates of the general elastic model are -within some confidence level- valid.

KEYWORDS: Poisson's ratio, elastic model, modulus of elasticity, modulus of rigidity, orthotropy.

\section{INTRODUCCIÓN}

Esta investigación presenta la metodología y los resultados de la determinación experimental de las características elásticas de dos especies de madera canadienses. Las especies estudiadas fueron Acer rubrum (Angiosperma) y Abies balsamea (Gimnosperma). Las características estu- diadas fueron: módulos de elasticidad, módulos de rigidez y coeficientes de Poisson. A partir de las características obtenidas empíricamente, se analizaron las propiedades de ortotropía de la matriz de constantes elásticas del modelo elástico general. 


\section{Modelo elástico general}

El comportamiento elástico de la madera es caracterizado por la ecuación constitutiva que expresa la ley de Hooke generalizada para un sólido elástico que posee simetrías materiales de tipo ortotrópico. El modelo elástico general del comportamiento elástico de la madera, a partir de la ley general de comportamiento elástico es:

$$
\varepsilon_{i j}=S_{i j k l} \sigma_{k l}
$$

Donde:

$\varepsilon_{i j}=$ Tensor de deformaciones.

$S i_{j k l}=$ Tensor de constantes elásticas.

$\sigma_{k l}=$ Tensor de esfuerzos.

con $i, j, k, l \in\{1,2,3\}$ por convención de índices repetidos.

Para el caso de la madera, idealizada como un sólido elástico, macroscópicamente homogéneo, de medio continuo y con simetrías materiales y elásticas de tipo ortotrópico, esta ley de comportamiento, se admite y se escribe con notación reducida de índices, como:

$$
\left[\begin{array}{c}
\varepsilon_{1} \\
\varepsilon_{2} \\
\varepsilon_{3} \\
\varepsilon_{4} \\
\varepsilon_{5} \\
\varepsilon_{6}
\end{array}\right]=\left[\begin{array}{cccccc}
S_{11} & S_{12} & S_{13} & 0 & 0 & 0 \\
S_{21} & S_{22} & S_{23} & 0 & 0 & 0 \\
S_{31} & S_{32} & S_{33} & 0 & 0 & 0 \\
0 & 0 & 0 & S_{44} & 0 & 0 \\
0 & 0 & 0 & 0 & S_{55} & 0 \\
0 & 0 & 0 & 0 & 0 & S_{66}
\end{array}\right]\left[\begin{array}{c}
\sigma_{1} \\
\sigma_{2} \\
\sigma_{3} \\
\tau_{4} \\
\tau_{5} \\
\tau_{6}
\end{array}\right]
$$

Con las simetrías:

$S_{12}=S_{21}$

$S_{13}=S_{31}$

$S_{23}=S_{32}$

Donde:

$\varepsilon=$ Deformación unitaria

$\sigma=$ Esfuerzo normal

$\tau=$ Esfuerzo cortante

Las constantes de elasticidad $S i j$ se pueden expresar en términos de las características elásticas de la madera en el sistema de coordenadas
$\{1,2,3\}:\{R, T, L\}$, como:

$$
\begin{aligned}
& S_{11}=1 / E_{R} \\
& S_{22}=1 / E_{T} \\
& S_{33}=1 / E_{L} \\
& S_{44}=1 / G_{T L} \\
& S_{55}=1 / G_{L R} \\
& S_{66}=1 / G_{R T} \\
& S_{12}=-v_{T R} / E_{T}=S_{21}=-v_{R T} / E_{R} \\
& S_{23}=-v_{L T} / E_{L}=S_{32}=-v_{T L} / E_{T} \\
& S_{13}=-v_{L R} / E_{L}=S_{31}=-v_{R L} / E_{R}
\end{aligned}
$$

Donde:

$R=$ Dirección radial

$T=$ Dirección tangencial

$L=$ Dirección longitudinal

$E=$ Módulo de elasticidad

$G=$ Módulo de rigidez

$v=$ Coeficiente de Poisson

Empleando las ecuaciones precedentes y aplicando las hipótesis simplificadoras de elasticidad, homogeneidad, medio continuo y simetrías materiales y elásticas ortotrópicas, la ley de comportamiento elástico de la madera se escribe en términos de las características elásticas de la madera como:

$$
\left[\begin{array}{c}
\varepsilon_{R} \\
\varepsilon_{T} \\
\varepsilon_{L} \\
\varepsilon_{T L} \\
\varepsilon_{L R} \\
\varepsilon_{R T}
\end{array}\right]=\left[\begin{array}{cccccc}
\frac{1}{E_{R}} & \frac{-v_{T R}}{E_{T}} & \frac{-v_{L R}}{E_{L}} & 0 & 0 & 0 \\
\frac{-v_{R T}}{E_{R}} & \frac{1}{E_{T}} & \frac{-v_{L T}}{E_{L}} & 0 & 0 & 0 \\
\frac{-v_{R L}}{E_{R}} & \frac{-v_{T L}}{E_{T}} & \frac{1}{E_{L}} & 0 & 0 & 0 \\
0 & 0 & 0 & \frac{1}{G_{T L}} & 0 & 0 \\
0 & 0 & 0 & 0 & \frac{1}{G_{L R}} & 0 \\
0 & 0 & 0 & 0 & 0 & \frac{1}{G_{R T}}
\end{array}\right]\left[\begin{array}{c}
\sigma_{R} \\
\sigma_{T} \\
\sigma_{L} \\
\tau_{T L} \\
\tau_{L R} \\
\tau_{T R}
\end{array}\right]
$$

y con las simetrías:

$-v_{T R} / E_{T}=-v_{R T} / E_{R}$

$-v_{L T} / E_{L}=-v_{T L} / E_{T}$

$-v_{L R} / E_{L}=-v_{R L} / E_{R}$ 


\section{Bases de datos experimentales}

Los datos de Hearmon (1948), Bodig y Goodman (1973) y Guitard y El Amri (1987) forman las 3 bases de datos de referencia reconocidas mundialmente. Hearmon recopiló datos para 18 maderas de especies angiospermas y 11 gimnospermas. Bodig y Goodman presentaron datos de 58 maderas de especies angiospermas y 71 gimnospermas. Guitard y El Amri recopilaron información de 43 maderas de especies angiospermas y 37 gimnospermas.

Por otra parte, distintos autores han presentado recientemente resultados experimentales y de modelización de características elásticas de maderas:

Bucur y Archer (1984) utilizaron un método de ultrasonido para determinar los módulos de elasticidad y de rigidez, así como los coeficientes de Poisson de 3 especies gimnospermas y 3 angiospermas. Bucur y Archer confirmaron la utilidad de las pruebas de ultrasonido en la determinación de las características elásticas de la madera. Igualmente los autores concluyen que la heterogeneidad y variación de la estructura anatómica de la madera limita la exactitud de las mediciones.

Sliker (1989), Sliker y Yu (1993) y Sliker et al. (1994), por medio de pruebas de compresión, tensión y cortante, determinaron los módulos de elasticidad, de rigidez y los coeficientes de Poisson para 25 maderas angiospermas y 5 maderas gimnospermas. Estos autores, confirmaron la utilidad de los sensores de deformación de resistencia eléctrica para medir deformaciones unitarias en la madera. Además los investigadores pusieron en evidencia el carácter ortotrópico de la madera y la variabilidad en los parámetros medidos al interior de cada especie.

Zink et al. (1997) utilizaron la técnica de correlación de imagen digital para medir coeficientes de Poisson en madera de Liriodendron tulipifera (angiosperma). Esta técnica permite visualizar el plano en estudio y de esta forma observar el fenómeno de deformación de manera más completa, en comparación con las medidas puntuales obtenidas utilizando sensores de deformación de resistencia eléctrica. Los investigadores notaron que el valor del coeficiente de Poisson no es constante durante el proceso carga-deformación del ensayo.
Liyu y Zhenyou (2004) estudiaron el carácter ortotrópico de la madera de Betula platyphylla Suk. (Angiosperma). Utilizando sensores de deformación de resistencia eléctrica, determinaron los módulos de elasticidad y rigidez, así como los módulos de Poisson correspondientes. En sus conclusiones, Liyu y Zhenyou comentan la influencia de la capa de adhesivo en el funcionamiento de los sensores de deformación. Igualmente los investigadores confirman las propiedades ortotrópicas de la madera.

Mascia (2003) y Mascia y Lahr (2006) realizaron pruebas de compresión en 2 maderas angiospermas y 2 especies gimnospermas. Los investigadores concluyeron que las predicciones de las componentes del tensor constitutivo del modelo elástico de la madera, son congruentes con los valores experimentales de la madera considerada como un material ortotrópico y de medio continuo. Igualmente los autores hacen notar que la variación angular en la dirección longitudinal de la madera influye en los valores de los componentes del tensor constitutivo del modelo elástico de la madera.

Keunecke et al. (2008) estudiaron el comportamiento elástico tridimensional de la madera. Los autores aplicaron pruebas de tensión en madera de Taxus baccata L. (angiosperma) y Picea abies (L.) Karst. (Gimnosperma). Los investigadores determinaron las 12 características elásticas de la madera necesarias para configurar la matriz de constantes elásticas. Entre sus principales conclusiones, Keunecke y colaboradores confirmaron el comportamiento ortotrópico de la madera de las especies estudiadas.

Laghdir et al. (2008) desarrollaron una técnica para determinar el tensor de elasticidad tridimensional de la madera aplicado al modelado de esfuerzos de secado. Los investigadores trabajaron con la madera de Picea mariana (Mill.) B.S.P. (gimnosperma). Los analistas validaron una técnica con un sensor de deformaciones reutilizable.

Niemz y Caduff (2008) determinaron para la madera de Picea abies (L.) Karst, los tres módulos de elasticidad y los seis coeficientes de Poisson utilizando un sensor de desplazamiento incremental en pruebas de tensión. Los autores validaron este método para la obtención de características elásticas de la madera. 
Es importante remarcar que los diferentes autores consultados, han trabajado con el paradigma del modelo elástico general, el cual considera a la madera como un material sólido, elástico, macroscópicamente homogéneo, de medio continuo y con una ortotropía material y elástica de tipo cilíndrica.

\section{Modelos de predicción}

Sliker y Yu (1993) y Sliker et al. (1994) propusieron modelos de predicción lineales para estimar características elásticas de maderas angiospermas, empleando como variable independiente la densidad del material. Las pruebas realizadas en tensión utilizaron probetas recortadas de placas. Para las pruebas de compresión, los autores utilizaron probetas compuestas de cubos de madera. El contenido de humedad fue de $12 \%$.

Mackenzie-Helnwein et al. (2005) realizaron pruebas en compresión y tensión biaxiales y mixtas de maderas de gimnospermas. A partir de sus resultados, los investigadores proponen modelos de predicción lineales para módulos de elasticidad, de rigidez y para coeficientes de Poisson. La característica de referencia de la madera fue su densidad. El contenido de humedad fue de $12 \%$.

Hernández-Maldonado (2010) estableció modelos estadísticos para predecir teóricamente valores de las características elásticas de la madera, a partir de un parámetro simple e intrínseco de la madera como es la densidad. Para seleccionar el tipo de regresión que será el modelo de predicción, realizó un estudio comparativo entre varios modelos estadísticos. Los modelos analizados fueron regresiones simples (lineales y exponenciales), múltiples y polinomiales. El modelo estadístico seleccionado fue del tipo:

$$
\mathrm{CE}=a \rho
$$

Donde:

$C E=$ Característica elástica

$\rho=$ Densidad de la madera $\left(\mathrm{g} / \mathrm{cm}^{3}\right)$

$a=$ Constante particular a cada grupo taxonómico y característica elástica
A manera de síntesis, se puede decir que el modelo elástico de la madera es aplicado de manera generalizada en las ciencias e ingeniería de la madera. En el mismo contexto, los datos experimentales de las características elásticas de la madera han sido calculados para especies con una amplia diversidad en los protocolos experimentales para su determinación. A excepción de la información contenida en las bases de datos, la información está dispersa en libros, tesis y artículos científicos. Esta problemática sugiere verificar experimentalmente la congruencia los valores existentes en la literatura.

\section{OBJETIVo}

Determinar experimentalmente los valores de las características elásticas módulos de elasticidad, módulos de rigidez y coeficientes de Poisson, de la madera de A. rubrum y de A. balsamea.

\section{MATERIALES Y MÉTODOS}

El material experimental consistió en madera de $A$. rubrum L. (Red Maple) y A. Balsamea (L.) Mill. (Balsam). Estas maderas están descritas entre otros autores, por Mullins y McKnight (1982), Lincoln (1986), Gilman y Watson (1993), Miller (1999), Spelter (2005) y Zerbe (2005).

Las dos especies de madera se seleccionaron con objeto de estudiar dos especies de divisiones taxonómicas distintas: angiospermas (A. rubrum) y gimnospermas ( $A$. Balsamea). De esta forma se obtuvieron dos muestras con densidades diferentes y anatomías distintas. Las dos maderas tienen características tecnológicas contrastables y son de uso generalizado en Canadá y en los Estados Unidos de América.

A partir de piezas de madera sólida, adquiridas en aserraderos localizados en la Provincia de New Brunswick, Canadá, se elaboraron dos grupos de probetas. Los especímenes fueron de pequeñas dimensiones y libres de particularidades de crecimiento, adaptando las especificaciones de la norma ASTM D143-94 (ASTM, 2009). La orientación de las aristas de las probetas fue paralela a las direcciones de ortotropía radial, tangencial y longitudinal 
del plano leñoso y sus dimensiones variaron de acuerdo con la combinación de su orientación y con la dirección de la solicitación (Fig. 1). Las probetas se prepararon en una cámara de acondicionamiento a un contenido de humedad de $9 \%$ para A. rubrum y de $10 \%$ para A. balsamea. Las configuraciones de las pruebas para una probeta de $A$. rubrum se ilustran en la figura 1.
Una serie de pruebas exploratorias de compresión, se realizaron con el objetivo de determinar el dominio elástico del proceso carga-deformación, para que las mediciones de las pruebas siguientes, se realizaran en un intervalo de aproximadamente $40 \%$ a $60 \%$ del límite elástico de la madera (Fig. 2). De esta manera, las pruebas adquirieron un carácter no destructivo. Utilizando probetas adiciona-

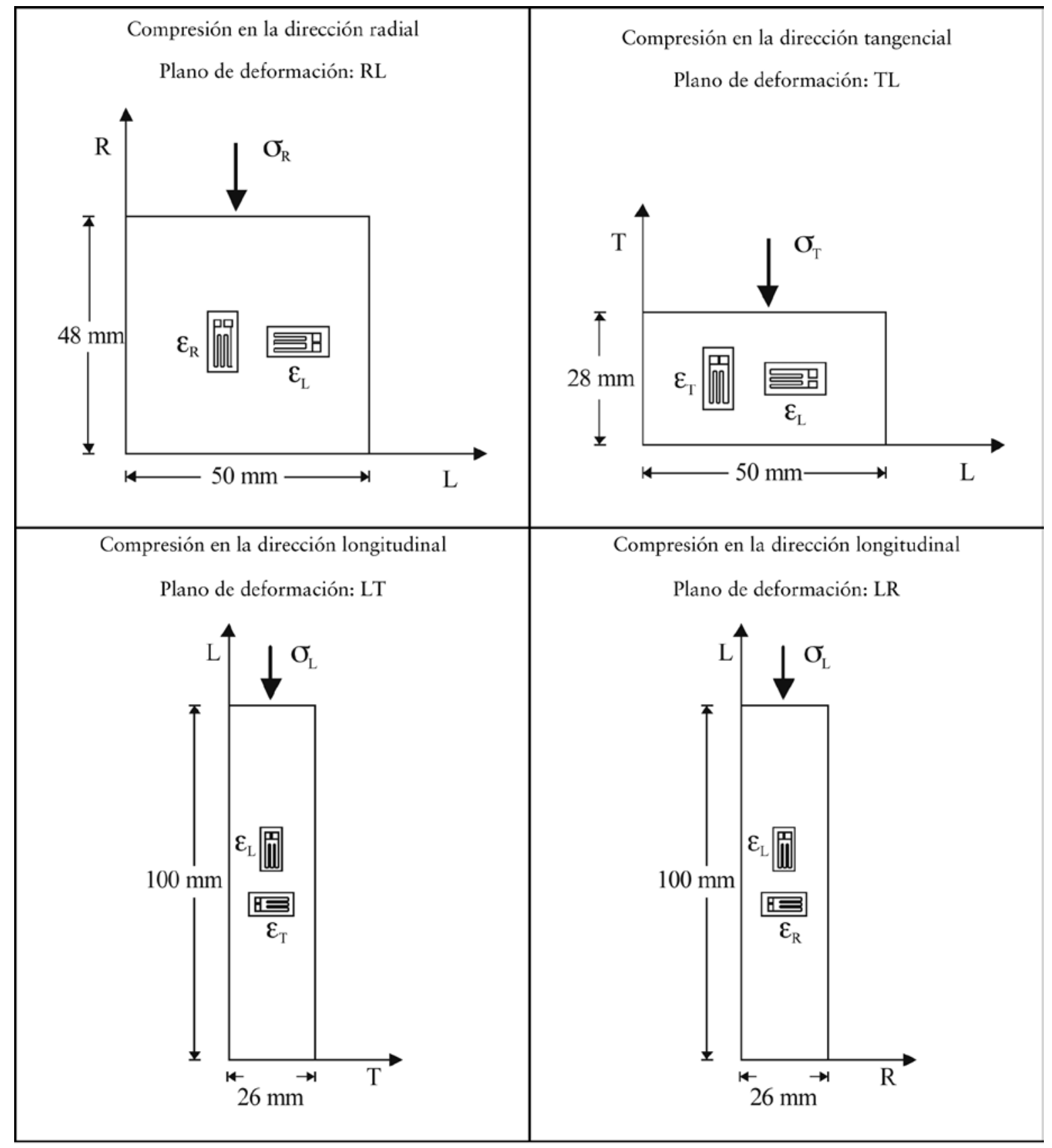

FIGURA 1. Configuración de las pruebas de compresión. 
les, se calcularon la densidad aparente ( $\rho$ ) y el contenido de humedad de la madera $(\mathrm{H})$ adaptando las especificaciones de la norma ASTM D143-94 (ASTM, 2009).

Las pruebas de compresión llevadas a la ruptura se realizaron en conjuntos de tres ejemplares, orientados en cada dirección de ortotropía de la madera: radial, tangencial y longitudinal. Cada conjunto correspondió a ensayos de compresión radial, tangencial y longitudinal.

En cada probeta se colocaron dos sensores de deformación de resistencia eléctrica en uno de los planos paralelos a la dirección de la solicitación (Fig. 1). Un sensor estuvo orientado de tal forma que midiera la deformación en la dirección colineal al sentido de la carga. El segundo sensor fue colocado de manera que captara las deformaciones en la dirección perpendicular a la dirección de la compresión.

Para los ensayos en compresión longitudinal, se colocaron cuatro sensores en los planos paralelos a la dirección de la carga y adyacentes entre sí. De esta forma se captaron simultáneamente las deformaciones en dos direcciones de anisotropía en planos paralelos a la carga.

Los parámetros que se midieron durante cada una de las pruebas de compresión fueron: la carga de compresión, el área resistente a la aplicación de la carga, y las deformaciones resultantes en las direcciones paralelas y perpendiculares, respecto a la orientación de la solicitación. Los módulos de elasticidad y los coeficientes de Poisson corres-

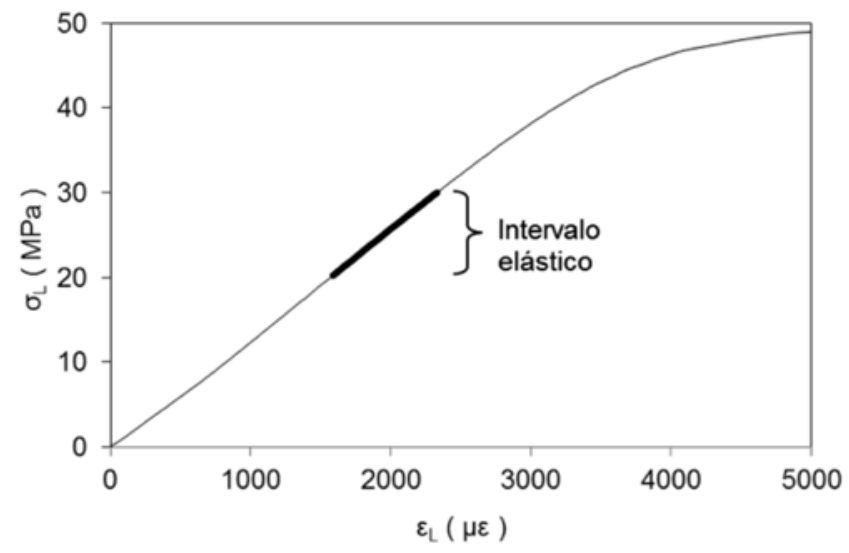

FIGURA 2. Diagrama esfuerzo-deformación. pondientes a cada prueba se calcularon a partir de las correlaciones estadísticas de los datos correspondientes al intervalo elástico de los diagramas esfuerzo-deformación (Fig. 2) y deformación-deformación (Fig. 3).

Para medir las deformaciones coaxial y normal a la dirección de la carga, se colocaron sensores de deformación de resistencia eléctrica CEA-06-500UW-350 de tecnología Vishay Micro-Measurements ${ }^{\circledR}$. Los sensores se posicionaron en los planos correspondientes y explicados en la figura 1.

La técnica de preparación y colocación de los sensores siguió los procedimientos especificados en las notas técnicas B-129-8 y TN-505-4 (Vishay Micro-Measurements, 1989 y 2009) y en el boletín B-127-14 (Vishay Micro-Measurements. 2005) de Vishay Micro-Measurements ${ }^{\circledR}$.

Para aplicar la solicitación en compresión, se utilizó una maquina universal de pruebas mecánicas MTS 810 de tecnología MTS Systems Corporation $®$.

La medición de las deformaciones se realizó conectando los sensores de deformación a un medidor de deformaciones P3 de tecnología Vishay Micro-Measurements ${ }^{\circledR}$.

Para la captura de datos de la carga aplicada a las probetas y de las deformaciones correspondientes, se conectaron la maquina universal y el medidor de deformaciones a un sistema de cómputo. El tratamiento posterior de datos se realizó utilizando un paquete comercial estadístico computarizado.

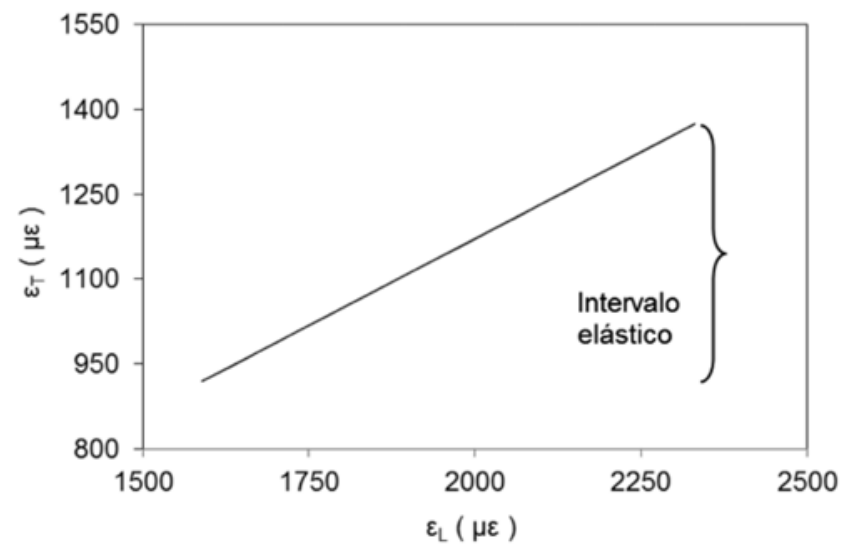

FIGURA 3. Diagrama deformación-deformación. 


\section{RESULTADOS}

La tabla 1 presenta los resultados de las pruebas de compresión. Los valores experimentales de $v_{R T}, v_{T R}$ no se presentan debido a dificultades en la adhesión de los sensores en el plano transversal (RT) a la dirección de la fibra de la madera, lo que podría alterar los datos. Los estadígrafos son: $x$ media aritmética, $\sigma$ desviación estándar y CV coeficiente de variación. Como valores de referencia, se presentan datos de Bodig y Goodman (1973).

En la tabla $1, G_{T L}, G_{L R}$ y $G_{R T}$ son los módulos de rigidez calculados a partir de los datos experimentales, con las relaciones propuestas por Lekhnitskii (1963) y verificadas por Mackenzie-Helnwein et al. (2005):

$$
G_{i j}=\frac{E_{i} E_{j}}{E_{i}-E_{j} 2 v_{i j} E_{j}}
$$

Donde:

$G_{i j}=$ Módulo de rigidez para el plano $i j(\mathrm{MPa})$.

$E_{i}=$ Módulo de elasticidad en la dirección $i(\mathrm{MPa})$.
$E_{j}=$ Módulo de elasticidad en la dirección $j(\mathrm{MPa})$.

$v_{i j}=$ Coeficiente de Poisson en el plano $i j$.

\section{DISCUSIÓN}

Las diferencias aritméticas entre los valores experimentales de las características elásticas obtenidas en el presente trabajo, comparados con los valores bibliográficos de Bodig y Goodman (1973) son importantes. No obstante, los datos aquí obtenidos para A. rubrum y A. balsamea, se localizan dentro del intervalo de dispersión de los datos bibliográficos; a excepción del valor del módulo de rigidez en al plano RT para A. balsamea, el cual se localiza por arriba de las predicciones. Además, para el caso de los coeficientes de Poisson, los resultados presentan tendencias similares a las de los módulos de elasticidad y de rigidez. Es decir, estos valores se distribuyen en regiones que giran alrededor de las estimaciones de trabajos anteriores. No obstante, las diferencias entre las características experimentales aquí obtenidas y los valores promedio de las bases de datos y sus coeficientes de variación son aceptables en relación con los intervalos de variación entre las características mecánicas de la madera estimadas por el

TABla 1. Módulos de elasticidad y rigidez obtenidos para la madera de A. rubrum y A. balsamea.

\begin{tabular}{|c|c|c|c|c|c|c|c|c|c|c|c|c|c|c|}
\hline & $\rho$ & $H$ & $E_{R}$ & $E_{T}$ & $E_{1}$ & $G_{T 1}$ & $G_{1 B}$ & $G_{R T}$ & & & & & & \\
\hline & $\mathrm{kg} / \mathrm{m}^{3}$ & $\%$ & $\mathrm{MPa}$ & $\mathrm{MPa}$ & $\mathrm{MPa}$ & $\mathrm{MPa}$ & $\mathrm{MPa}$ & $\mathrm{MPa}$ & $V_{R T}$ & $v_{T R}$ & $v_{R L}$ & $v_{L R}$ & $v_{T L}$ & $v_{L T}$ \\
\hline \multicolumn{15}{|c|}{ A. rubrum } \\
\hline$x$ & 651 & 9,0 & 1431 & 960 & 11879 & 826 & 1138 & 354 & - & - & 0,063 & 0,525 & 0,041 & 0,550 \\
\hline$\sigma$ & - & - & 230 & 85 & 1637 & 72 & 114 & 26 & - & - & 0,0056 & 0,0237 & 0,0022 & 0,0459 \\
\hline$C V$ & - & - & 0,16 & 0,09 & 0,14 & 0,09 & 0,10 & 0,07 & - & - & 0,09 & 0,05 & 0,05 & 0,08 \\
\hline \multicolumn{15}{|c|}{ A. rubrum (Bodig y Goodman, 1973) } \\
\hline$x$ & 500 & 11,4 & 1122 & 550 & 12480 & 639 & 869 & 212 & 0,733 & 0,360 & 0,037 & 0,412 & 0,023 & 0,531 \\
\hline \multicolumn{15}{|c|}{ A, balsamea } \\
\hline$x$ & 393 & 10,0 & 861 & 633 & 11617 & 575 & 745 & 261 & - & - & 0,025 & 0,451 & 0,022 & 0,464 \\
\hline$\sigma$ & - & - & 296 & 109 & 752 & 87 & 229 & 72 & - & - & 0,0056 & 0,0147 & 0,0055 & 0,0541 \\
\hline$C V$ & - & - & 0,34 & 0,17 & 0,06 & 0,15 & 0,31 & 0,27 & - & - & 0,22 & 0,03 & 0,25 & 0,12 \\
\hline \multicolumn{15}{|c|}{ A. balsamea (Bodig y Goodman, 1973) } \\
\hline$x$ & 340 & 12,0 & 743 & 421 & 9336 & 615 & 640 & 56 & 0,481 & 0,272 & 0,036 & 0,450 & 0,024 & 0,536 \\
\hline
\end{tabular}


Laboratorio de Productos Forestales de los Estados Unidos de América (FPL, 1999).

Por otra parte, la magnitud de la variación para $A$. balsamea, es del mismo orden que la de la variación presentada por Bucur y Rasolofosaon (1998), de 5\% a $15 \%$ para maderas de los géneros Quercus y Pinus. Para el caso de A. rubrum, la variación de los términos diagonales es más grande. Aún más, las diferencias aritméticas entre resultados bibliográficos y de laboratorio, son más evidentes, si se comparan los valores de las características elásticas para cada especie estudiada. Este resultado es particularmente notorio para los valores de los coeficientes de Poisson.

Respecto a la anisotropía de las características elásticas, las relaciones entre ellas son, para A. rubrum:

$$
\begin{gathered}
E_{L} \gg E_{R}>E_{T}: 11879 \gg 1431>960 \\
G_{L R} \gg G_{T L}>G_{R T}: 1138 \gg 826>354 \\
v_{R T}>v_{L T}>v_{L R} \approx v_{T R}>>v_{R L}>v_{T L}: 0,756>0,550>0,525 \\
\approx 0,388>>0,063>0,041
\end{gathered}
$$

y para A. balsamea:

$$
\begin{gathered}
E_{L} \gg E_{R}>E_{T}: 11617 \gg 861>633 \\
G_{L R} \gg G_{T L}>G_{R T}: 745 \gg 575>74 \\
v_{R T}>v_{L T}>v_{L R} \approx v_{T R}>>v_{R L}>v_{T L}: 0,456>0,464>0,451 \\
\approx 0,297>>0,025>0,022
\end{gathered}
$$

Las proporciones anteriores coinciden con las de Guitard (1987), a excepción de $v_{R T}$ que es un valor estimado, lo cual puede explicar que es menor que $v_{L T}$. Estos resultados confirman los requerimientos del modelo elástico general con respecto a las relaciones de anisotropía observadas en la madera.

Estos resultados sugieren una variabilidad en la magnitud de los parámetros de ingeniería, a partir de los cuales se calculan las constantes de las matrices de elasticidad de la madera. Es decir, los datos experimentales son congruentes pero denotan una variabilidad inherente al material. Estas discrepancias entre resultados pueden ser explicadas por los siguientes argumentos:
El origen de la variación sistémica en la magnitud de los parámetros de Ingeniería de la madera, puede ser atribuido a la variación de las características mecánicas que existe entre especies, al interior de una especie y según el espécimen de madera examinado. Igualmente, influye la localización en el fuste del árbol y las particularidades de crecimiento de la madera con la que están elaboradas las probetas (Mattheck y Kubler, 1997, Wodzicki, 2001 y Barnett y Jeronimidis, 2003).

Las diferencias entre los contenidos de humedad y las densidades de la madera influyen en los resultados (Brown et al. (1952), Dinwoodie (1981), Tsoumis (1991) y Forest Products Laboratory (1999).

Los datos derivados de los modelos de predicción propuestos por Hernández-Maldonado (2010), se refieren a madera con un contenido de humedad de $12 \%$. En contraste, el contenido de humedad de las probetas de este estudio fue de entre $9 \%$ y $10 \%$. Igualmente, las densidades presentadas por Bodig y Goodman (1973) son distintas a las densidades de la madera de A. rubrum y $A$. balsamea aquí encontradas.

Respecto a las experiencias realizadas, otra fuente de variación entre los resultados teóricos y experimentales son las diferentes configuraciones de los ensayos con los cuales se determinaron las características recopiladas para los modelos de predicción.

Los métodos de laboratorio referidos o utilizados por Hearmon (1948), Bodig y Goodman (1973) y Guitard y El Amri (1987), son variados y distintos a la configuración de las pruebas de compresión realizadas en esta investigación. Es decir, el tipo, la dirección y la velocidad de las solicitaciones aplicadas en los ensayos no fueron necesariamente las mismas. Lo que puede influir en la diferencia en magnitud de los resultados.

\section{Simetrías de las constantes elásticas}

Para verificar el requisito de simetría en la matriz de constantes elásticas del modelo elástico general, es necesario calcular la variación entre los términos no diagonales de la ecuación del modelo elástico general. 
TABla 2. Coeficientes de anisotropía de constantes de elasticidad.

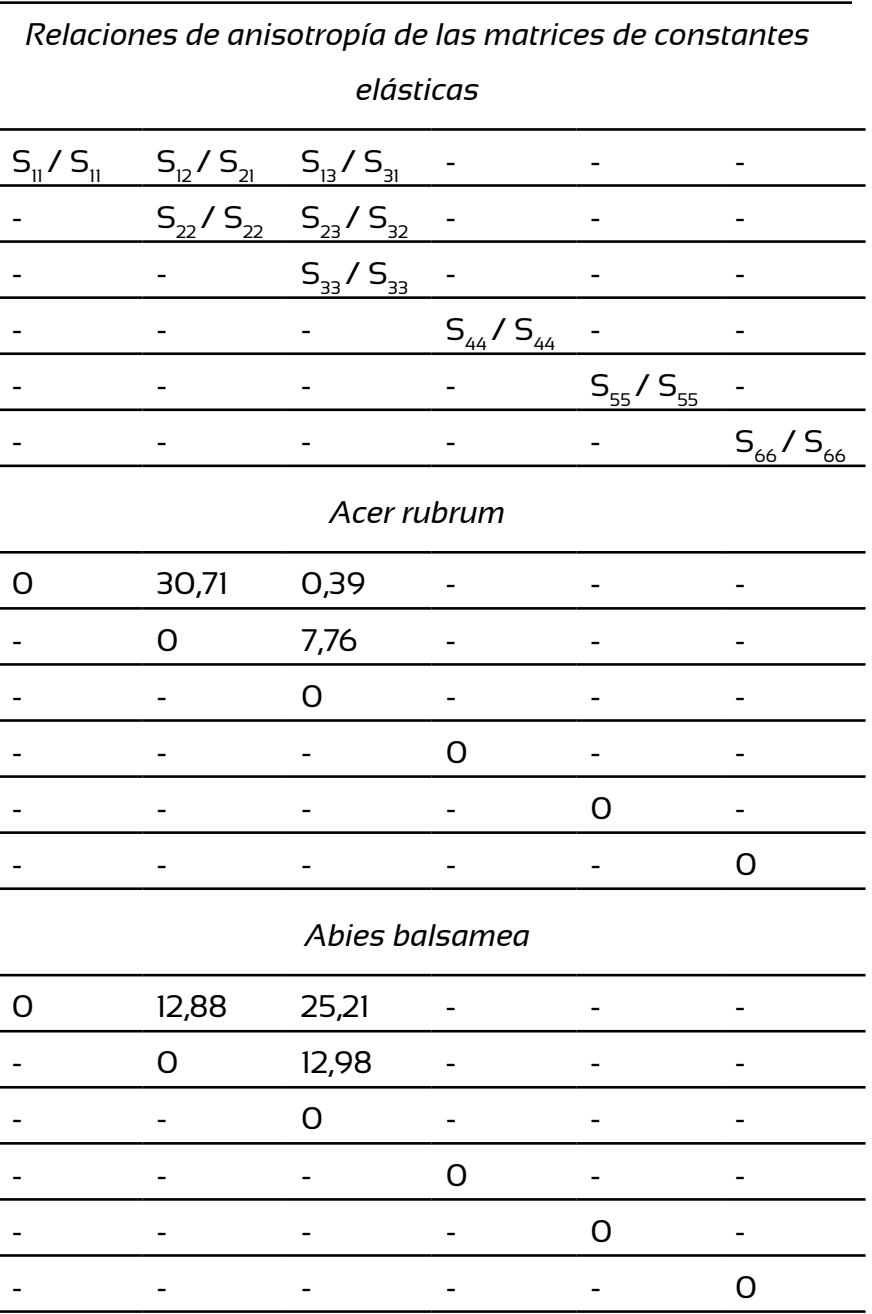

La variación en la simetría de las matrices de constantes elásticas está expresada con los coeficientes de anisotropía propuestos por Bucur y Rasolofosaon (1998):

$$
S_{i j} / S_{j i}=\left[\left(S_{i j}-S_{j i}\right) / S_{i j}\right] \times(100)
$$

Las matrices de constantes elásticas presentadas en la tabla 2, fueron determinadas a partir de los resultados experimentales para A. rubrum y A. balsamea. Es importante hacer notar que los términos no diagonales de las matrices de constantes elásticas están compuestos de los módulos de elasticidad y de los coeficientes de Poisson correspondientes al argumento de ortotropía del modelo elástico general.

De la observación de la tabla 2 se puede destacar que las variaciones para los tres componentes $\left(S_{12} / S_{21}, S_{13} / S_{31}\right.$ y $S_{23} / S_{32}$ ) de cada matriz son desiguales para cada especie. Es decir, existe una cierta tendencia en la amplitud de la variación al interior de cada grupo de valores analizados.

\section{CONCLUSIONES}

Se determinaron las características elásticas de las maderas de A. rubrum y de A. balsamea.

A partir de los resultados experimentales y del análisis comparativo con datos de otros estudios, se concluye lo siguiente: las características elásticas de A. rubrum y de $A$. balsamea son comparables con valores correspondientes de estas especies determinados en investigaciones recientes y con bases de datos publicados en la bibliografía.

El análisis de la variación de las características y de las constantes elásticas de la madera de A. rubrum y de $A$. balsamea demostró que los postulados de ortotropía del modelo elástico general son -con cierta precisión- válidos.

\section{RECONOCIMIENTOS}

La etapa experimental se realizó en el Centro de Ciencia y Tecnología de la Madera de la Facultad de Forestería y Manejo del Medio Ambiente, de la Universidad de New Brunswick, Canadá. Los trabajos de laboratorio fueron supervisados por los Profesores Ying Hei Chui y Meng Gong y por el Tecnólogo Dean McCarthy. La estancia de investigación del primer autor en Canadá fue patrocinada por el Consejo Nacional de Ciencia y Tecnología de México.

\section{REFERENCIAS}

ASTM (American Society for Testing and Materials International). 2009. D0143-94R07 Test Methods for Small Clear Specimens of Timber. Annual Book of ASTM Standards. ASTM Volume 04.10 Wood. EUA.

Barnett, J.R. y G. Jeronimidis. 2003. Wood quality and its biological basis. Blackwell Publishing. CRC Press. Inglaterra.

Bodig, J. y J.R. Goodman. 1973. Prediction of elastic parameters for wood. Wood Science. 5(4):249-264. 
Brown, H.P., A.J. Panshin y C.C. Forsaith. 1952. Texbook of wood technology. Volume II. McGraw-Hill. EUA.

Bucur, V. y R.R. Archer. 1984. Elastic constants for wood by an ultrasonic method. Wood Science and Technology. 18:255-265.

Bucur, V. y P.N.J. Rasolofosaon. 1998. Dynamic elastic anisotropy and nonlinearity in wood and rock. Ultrasonics. 36:813-824.

Dinwoodie, J.M. 1981. Timber, its nature and behaviour. Van Nostrand-Reinhold. EUA.

Gilman, E.F. y D.G. Watson. 1993. A. Rubrum. Red maple. Fact sheet ST-41. Environmental Horticulture Department, Florida Cooperative Extension Service, Institute of Food and Agricultural Sciences, University of Florida. EUA.

Forest Products Laboratory. 1999. Wood handbook-Wood as an engineering material. Gen. Tech. Rep. FPL-GTR-113. USDA Forest Service. Forest Products Laboratory. Madison, Wi. $508 \mathrm{p}$.

Forest Products Laboratory. 1953. Hardwood log grades for standard lumber. Rept. D1737. USDA Forest Service. Forest Products Laboratory. Madison, Wi. 66 p.

Guitard, D. 1987. Mécanique du matériau bois et composites. Cepadues-Editions. Francia.

Guitard, D. y F. El Amri. 1987. Modèles prévisionnels de comportement élastique tradimensionnel pour les bois feuillus et les bois résineux. Annales des Sciences Forestières. INRA 45(3):335-358.

Haygreen, J.G. y J.L. Bowyer. 1996. Forest products and wood science, an introduction. Iowa State University Press. EUA.

Hearmon, R.F.S. 1948. The elasticity of wood and plywood. Department of Scientific and Industrial Research. Forest Products Research. Special Report No. 7. His Majesty's Stationery Office. Inglaterra.

Hernández-Maldonado, S.A. 2010. Comportamiento elástico de la madera. Teoría y aplicaciones. Tesis de Maestría en Ciencias y Tecnología de la Madera. Universidad Michoacana de San Nicolás de Hidalgo.

Keunecke, D., S. Hering y P. Niems. 2008. Three-dimensional elastic behaviour of common yew and Norway spruce. Wood Science and Technology 42:633-647.
Laghdir, A., Y. Fortin, C.M. De la Cruz y R.E. Hernández. 2008. Development of a technique to determine the 3D elasticity tensor of wood as applied to drying stress modeling. Maderas. Ciencia y tecnología 10(1):35-44.

Lekhnitskii, S.G. 1963. Theory of Elasticity of an Anisotropic Elastic Body. Holden-Day. EUA.

Lincoln, W.A. 1986. World woods in color. Linden Publishing. EUA.

Liyu, W. y L. Zhenyou. 2004. Twelve elastic constants of Betula platyphylla Suk. Forestry Studies in China 6(1):37-41.

Mackenzie-Helnwein, P., J. Eberhardsteiner y H.A. Mang. 2005. Rate-independent behavior of biaxially stressed Wood: Experimental observations and constitutive modeling as an orthotropic two-surface elasto-plastic material. Holzforschung 59:311-321.

Mascia, N.T. 2003. Concerning the elastic orthotropic model applied to wood elastic properties. Modelo elástico ortotrópico aplicado a la madera. Maderas. Ciencia y tecnología. 5(1):3-19.

Mascia, N.T. y F.A.R. Lahr. 2006. Remarks on orthotropic elastic models applied to wood. Materials Research. 9(3):301-310.

Mattheck, C. y H. Kubler. 1997. Wood - The Internal Optimization of Trees. Springer-Verlag. Alemania.

Miller, R.B. 1999. Characteristics and availability of commercially important woods. In: Forest Products Laboratory. Wood handbook-Wood as an engineering material. Gen. Tech. Rep. FPL-GTR-113. USDA Forest Service. Forest Products Laboratory. Madison, Wi. p:2-2 a 2-45.

Mullins, E.J. y T.S. McKnight. 1982. Third Edition. Canadian woods. Their properties and uses. University of Toronto Press. Canadian Forestry Service, Environment Canada. Canadian Government Publishing Centre, Supply and Services Canada.

Niemz, P. y D. Caduff. 2008. Untersuchungen zur bestimmung der Poissonschen konstanten an fichtenholz. European Journal of Wood and Wood Products 66:1-4.

Sliker, A. 1989. Measurement of the smaller Poisson's ratios and relates compliances for wood. Wood and Fiber Science 21(3):252-262. 
Sliker, A. y Y. Yu. 1993. Elastic constants for hardwoods measured from plate and tension tests. Wood and Fiber Science 25(1):8-22.

Sliker, A.; Y. Yu, T. Weigel y W. Zangh 1994. Orthotropic elastic constants for eastern hardwood species. Wood and Fiber Science 26(1):107-121.

Spelter, H. 2005. Red and silver maples - Resource availability, utilization, and costs. In: R.J. Ross y J.R Erickson, eds. Undervalued hardwoods for engineering materials and components. Forest Products Society. Northern Initiatives. EUA.

Tsoumis, G. 1991. Science and Technology of Wood. Structure, properties, utilization. Editorial Van Nostrand Reinhold. USA.
Wodzicki, T.J. 2001. Natural factors affecting wood structure. Wood Science and Technology 35:5-26.

Zerbe, J.I. 2005. Basic properties of undervalued hardwoods. In: R.J. Ross y J.R. Erickson, eds. Undervalued Hardwoods for Engineering Materials and Components. Forest Products Society. Northern Initiatives. EUA.

Zink, A.G., R.B. Hanna y J.W. Stelmokas. 1997. Measurement of Poisson's ratios for yellow-poplar. Forest Products Journal 47(3):78-80.

Manuscrito recibido el 18 de febrero de 2013.

Aceptado el 23 de mayo de 2014.

Este documento se debe citar como:

Hernández-Maldonado, S.A. y J.R. Sotomayor-Castellanos. 2014. Comportamiento elástico de la madera de Acer rubrum y de Abies balsamea. Madera y Bosques 20(3):113-123. 